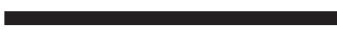

Armstrong, N., Barker, A., \& McManus, A. (2017). Muscle metabolism during exercise. In N. Armstrong, W. van Mechelen (Eds), Oxford textbook of children's sport and exercise medicine (pp. 69-87). Oxford University Press

Bangsbo (1998). Entrenamiento de la condición física em el fútbol ( $2^{a}$ Ed.). Editorial Paidotribo

Elferink-Gemser, M., Visscher, C., Richart, H., \& Lemmink, K. (2004). Development of the tactical skills inventory for sports. Perceptual and Motor Skills, 99, 883-895.

Ellison, P.T., Reiches, M.W., Shattuck-Faegre, H., Breakey. A., Konecna, M., Urlacher, S., \& Wobber, V. (2012). Puberty as a life history transition. Annals of Human Biology, 39, 352-360. doi:10.3109/03014460.2012693199

Guilherme Oliveira, J., Garganta, J., Graça, A., \& Seabra, A. (2015). Influence of non-preferred foot technical training in reducing lower limbs functional asymmetry among young football players, Journal of Sports Sciences, 33, 17901798. doi:10.1080/02640414.2015.1012100.

Haugaasen, M., \& Jordet, G. (2012). Developing football expertise: A football-specific research review. International Review of Sport and Exercise Psychology, 5, 177-201. European Club Association. (2012). Report on youth academies in Europe. European Club Association.

Kannekens, R., Elferink-Gemser, M., Post, W., \& Visscher, C. (2009). Self-assessed tactical skills in elite youth soccer players: a longitudinal study. Perceptual Motor Skills. 109, 459-472

Leyhr, D., Kelava, A., Raabe, J., \& Honer, O. (2018). Longitudinal motor performance development in early adolescence and its relationship to adult success: An 8-year prospective study of highly talented soccer players. PLoS One, 13, e0196324. doi:10.1371/journal.pone.0196324 Lloyd, R. S., Cronin, J. B., Faigenbaum, A. D., Haff, G. G. Howard, R., Kraemer, W. J., Micheli, L. J., Myer, G. D., \& Oliver, J. L. (2016). National Strength and Conditioning Association position statement on long-term athletic development. Journal of Strength Conditioning and Research, 30, 1491-1509.
Pereira, M. Tavares, F. Santos, A., \& Graça, A. (in press). Adaptação transcultural do Questionário de Competências Táticas nos Jogos Desportivos Coletivos. Revista Portuguesa de Ciências do Desporto.

Rebelo, A., Brito, J., Seabra, A., Oliveira, J., \& Krustrup, P. (2014). Physical match performance of youth footbal p. of Sport Science, 14(Suppl.1), S148-S156. doi:10.1080/17 461391.2012.664171

Rees, T., Hardy, L., Güllich, A., Abernethy, B., Côté, J Woodman, T., Montgomery, H., Laing, S., \& Warr, C. (2016). The great British medalists project: A review of current knowledge on the development of the world's best sporting talent. Sports Medicine, 46, 1041-1058.

Ribeiro, J., Davids, K., Silva, P., Coutinho, P., Barreira, D. \& Garganta, J. (2021). Talent development in sport requires athlete enrichment: Contemporary insights from a nonlinear pedagogy and the athletic skills model. Sports Medicine, 51(6), 1115-1122. doi:10.1007/s40279-02101437-6.

Schreiner, P. (2000). Coordination agility \& speed training for soccer. Reedswain Incorporated

Sgrò, F., Bracco, S., Pignato, S., \& Lipoma, M. (2018). Small-sided games and technical skills in soccer training: Systematic review and implications for sport and physial education practitioners. Journal of Sports Sciences, 6 9-19.

Wilson, R. S., James, R. S., David, G., Hermann, E., Moran O. J., Niehaus, A. C.. Hunter, A., Thake, D., \& Smith, M. D. (2016). Multivariate analyses of individual variation in soccer skill as a tool for talent identification and deelopment: utilising evolutionary theory in sports science. Journal of Sports Sciences, 34, 2074-2086. doi:10.1080/0 2640414.2016.1151544
AUTORES:

Ricardo Fernandes ${ }^{1,2}$

No início era assim.

Filipa Cardoso ${ }^{1}$

Unidade na diversidade.

Polo aquático

Manoel Rios

Sofia Canossa ${ }^{1}$

Centro de Investigação, Formação, Intervenção e Inovação em Despo to (CIFI ${ }^{2}$ ), Faculdade de Desporto,

Universidade do Porto, Portugal

¿Laboratório de Biomecânica do Porto (LABIOMEP), Universidade do Porto,

Portugal

https://doi.org/10.5628/rpcd.21.S2.109

\section{DOMÍNIO BIOLÓGICO}

0 jogo de polo aquático integra as atividades desportivas contemporâneas, encontrando-se difundido por todo o mundo. Aliado ao seu passado histórico (é um desporto pertencente ao programa dos Jogos Olímpicos desde a edição de Paris'1900), pode constituir-se como um forte atrativo de prática aquática em diversas instituições públicas e privadas. No entanto, muito embora a sua expansão nas últimas décadas e a existência de um número crescente de jovens a praticá-lo, existe a necessidade do estudo mais aprofundado deste jogo desportivo no sentido de melhor se compreender a sua complexidade e utilizar o conhecimento daí decorrente para promover maior qualidade e eficácia nos processos de ensino e treino (Canossa et al., 2009). De facto, embora já se saiba que os jogadores realizam esforços intermitentes, alternando fases de elevada intensidade com outras de recuperação, utilizando ações acíclicas, variáveis e abertas (e.g. Botonis et al., 2019), o conhecimento na área ainda está bastante aquém do desejado, nomeadamente nos escalões de formação.

Como se poderá constatar no quadro 1 , na primeira avaliação do INEX, os jogadores mais velhos (coorte 3 ) eram mais pesados $\left(F=3.78, p<.05, \mathrm{p}^{2}=.91\right.$ ), com mais massa isenta de gordura $\left(F=8.64, p<.001, \mathrm{p \eta}^{2}=.18\right)$ e maturacionalmente mais avançados relativamente aos seu pares $\left(F=28.35, p<.001, \mathrm{p \eta}^{2}=.43\right)$. Estas diferenças podem ser explicadas pelo crescimento físico e maturação sexual dos adolescentes, mas também pela influência do treino e competição regular (Aleksandrović et al., 2007). De facto, o treino regular tem o potencial de influenciar favoravelmente a composição corporal, aumentando a densidade mineral óssea e a musculatura esquelética, e diminuindo a gordura 
(especialmente em rapazes). Inesperadamente, os sujeitos da coorte 2 eram mais altos ( $F$ $\left.=6.01, p<.05, \mathrm{p \eta}^{2}=.14\right)$, com maior envergadura $\left(F=6.10, p<.05, \mathrm{p \eta}^{2}=.14\right)$ e diâmetro palmar (transversal e longitudinal: $F=8.82, p<.001, \mathrm{p \eta}^{2}=.19$ e $F=9.69, p<.001, \mathrm{pn}^{2}$ $=.20$ ), o que pode ser explicado pelas diferenças do $\mathrm{n}$ amostral nas coortes 2 e 3 . Este resultado também pode ser atribuído a fatores genéticos pois a baixa estatura de alguns jogadores pode ser uma característica familiar já que crianças e jovens baixos tendem a te pais baixos (Malina, 2009). Relativamente à massa gorda, não se verificaram diferenças entre as coortes estudadas.

O lançamento da bola medicinal em posição sentado foi outra variável onde se encontraram diferenças entre os grupos etários em estudo $\left(F=15.15, p<.001, \mathrm{pn}^{2}=.28\right)$, as quais poderão não depender apenas do aumento da força devido à maturação biológica, mas também devido ao efeito da carga de treino. De facto, o desenvolvimento específico da força muscula do tronco e dos membros superiores é um dos focos do processo de treino no polo aquático o qual exige uma deslocação eficiente no meio aquático (através das técnicas de nado e de remada), assim como uma técnica de remate evoluída (Keiner et al., 2020).

QUADRO 1. Resultados dos jogadores de polo aquático (idade, experiência de treino e domínio biológico), por coorte, no baseline

\begin{tabular}{|c|c|c|c|c|c|}
\hline \multirow{3}{*}{ DOMÍNIO BIOLÓGICO } & COORTE 1 (C1) & COORTE 2 (C2) & COORTE 3 (C3) & \multirow{3}{*}{$F\left(\mathrm{pn}^{2}\right)^{2}$} & \multirow{3}{*}{ CONTRASTE $^{3}$} \\
\hline & $(n=28)$ & $(n=41)$ & $(n=11)$ & & \\
\hline & $M \pm D P^{1}$ & $M \pm D P$ & $M \pm D P$ & & \\
\hline Idade (anos) & $12.55 \pm 0.28$ & $13.53 \pm 0.26$ & $14.14 \pm 0.16$ & $188.80^{4 \times(}(83)$ & $\mathrm{C} 1<\mathrm{C} 2<\mathrm{C} 3$ \\
\hline Experiência de treino (anos) & $2.33 \pm 0.33$ & $2.54 \pm 0.32$ & $3.00 \pm 0.84$ & $0.41 \mathrm{~ns}(.01)$ & $\mathrm{C} 1<\mathrm{C} 2<\mathrm{C} 3$ \\
\hline \multicolumn{6}{|l|}{$\begin{array}{l}\text { ANTROPOMETRIA E } \\
\text { COMPOSIÇÃO CORPORAL }\end{array}$} \\
\hline Altura $(\mathrm{cm})$ & $156.33 \pm 8.21$ & $163.12 \pm 8.96$ & $162.89 \pm 3.77$ & $6.01 \mathrm{~ns}(.14)$ & $\mathrm{C} 1<\mathrm{C} 2>\mathrm{C} 3$ \\
\hline Peso $(\mathrm{kg})$ & $49.76 \pm 10.46$ & $54.23 \pm 9.04$ & $58.50 \pm 7.87$ & $3.78 n s(.91)$ & $\mathrm{C} 1<\mathrm{C} 2<\mathrm{C} 3$ \\
\hline Envergadura $(\mathrm{cm})$ & $159.64 \pm 9.27$ & $167.12 \pm 8.88$ & $164.34 \pm 5.37$ & $6.10 \mathrm{~ns}(.14)$ & $\mathrm{C} 1<\mathrm{C} 2>\mathrm{C} 3$ \\
\hline Diâmetro palmar transversal (cm) & $18.95 \pm 1.53$ & $20.48 \pm 1.57$ & $20.30 \pm 1.03$ & $8.82^{2 * \star}(19)$ & $\mathrm{C} 1<\mathrm{C} 2, \mathrm{C} 3$ \\
\hline Diâmetro palmar longitudinal (cm) & $16.90 \pm 1.13$ & $18.05 \pm 1.11$ & $17.87 \pm 0.65$ & $9.69^{* \star *}(.20)$ & $\mathrm{C} 1<\mathrm{C} 2>\mathrm{C} 3$ \\
\hline Massa gorda (kg) & $10.97 \pm 4.42$ & $9.70 \pm 3.10$ & $11.80 \pm 6.06$ & $1.49 n s(.03)$ & $\mathrm{C} 1>\mathrm{C} 2<\mathrm{C} 3>\mathrm{Cl}$ \\
\hline Massa isenta de gordura $(\mathrm{kg})$ & $38.80 \pm 6.89$ & $44.60 \pm 6.70$ & $46.78 \pm 4.64$ & $8.64^{4 * *(.18)}$ & $\mathrm{C} 1<\mathrm{C} 2<\mathrm{C} 3$ \\
\hline
\end{tabular}
baseline (cont.)

\begin{tabular}{|c|c|c|c|c|c|}
\hline \multirow{3}{*}{ DOMÍNIO BIOLÓGICO } & COORTE 1 (C1) & COORTE 2 (C2) & COORTE 3 (C3) & \multirow{3}{*}{$F\left(\mathrm{pn}^{2}\right)^{2}$} & \multirow{3}{*}{ CONTRASTE ${ }^{3}$} \\
\hline & $(n=27)$ & $(n=41)$ & $(n=11)$ & & \\
\hline & $M \pm D P^{1}$ & $M \pm D P$ & $M \pm D P$ & & \\
\hline \multicolumn{6}{|l|}{ MATURAÇÃO BIOLÓGICA } \\
\hline Offset maturacional (anos) & $-1.13 \pm 0.63$ & $-0.13 \pm 0.68$ & $0.32 \pm 0.41$ & $28.35^{\star \star \star *}(.43)$ & $\mathrm{C} 1, \mathrm{C} 2<\mathrm{C} 3$ \\
\hline \multicolumn{6}{|l|}{ DESEMPENHO Fí́SICO } \\
\hline Preensão manual (kg') & $21.51 \pm 7.29$ & $26.24 \pm 8.01$ & $29.12 \pm 13.17$ & $3.96(.09)$ & $\mathrm{C} 1<\mathrm{C} 2<\mathrm{C} 3$ \\
\hline Sprint 5m (s) & $1.31 \pm 0.24$ & $1.14 \pm 0.27$ & $1.38 \pm 0.11$ & $5.94 n s(.13)$ & $\mathrm{C} 1>\mathrm{C} 2<\mathrm{C} 3>\mathrm{C} 1$ \\
\hline Sprint 20m (s) & $3.90 \pm 0.39$ & $3.45 \pm 0.64$ & $3.78 \pm 0.24$ & 6.73ns(.15) & $\begin{array}{l}\mathrm{C} 1>\mathrm{C} 2, \mathrm{C} 3 ; \\
\mathrm{C} 2<\mathrm{C} 3\end{array}$ \\
\hline Abdominais (repetiçōes) & $32.18 \pm 7.13$ & $37.67 \pm 5.99$ & $38.17 \pm 5.71$ & $6.00 n s(.16)$ & $\mathrm{C} 1<\mathrm{C} 2<\mathrm{C} 3$ \\
\hline $\begin{array}{l}\text { Salto vertical sem } \\
\text { contramovimento (cm) }\end{array}$ & $21.14 \pm 4.83$ & $24.96 \pm 6.71$ & $22.81 \pm 3.05$ & $3.74 n s(.09)$ & $\mathrm{C} 1<\mathrm{C} 2 ; \mathrm{C} 3<\mathrm{C} 5$ \\
\hline $\begin{array}{l}\text { Salto vertical com } \\
\text { contramovimento (cm) }\end{array}$ & $22.73 \pm 4.95$ & $26.35 \pm 6.23$ & $24.65 \pm 3.39$ & $3.62 n s(.09)$ & $\begin{array}{c}\mathrm{Cl}<\mathrm{C} 2>\mathrm{C} 3 ; \\
\mathrm{C} 3>\mathrm{Cl}\end{array}$ \\
\hline $\begin{array}{l}\text { Lançamento sentado da bola } \\
\text { medicinal }(\mathrm{m})\end{array}$ & $3.03 \pm 0.49$ & $3.61 \pm 0.49$ & $3.78 \pm 0.42$ & $15.15^{\star \star \star} \times(28)$ & $\mathrm{C} 1<\mathrm{C} 2<\mathrm{C} 3$ \\
\hline T-test (s) & $11.57 \pm 0.93$ & $10.86 \pm 0.79$ & $10.31 \pm 0.99$ & $10.13^{\star \star \star \star}(.21)$ & $\mathrm{C} 1>\mathrm{C} 2, \mathrm{C} 3$ \\
\hline Yo-Yo IRI (m) & $361.43 \pm 239.62$ & $546.00 \pm 257.16$ & $646.67 \pm 340.20$ & $6.26 n s(.14)$ & $\mathrm{C} 1<\mathrm{C} 2<\mathrm{C} 3$ \\
\hline
\end{tabular}

\section{DOMINIO DAS HABILIDADES ESPECIFICAS/}

\section{PERCETIVO-COGNITIVAS}

Sendo o polo aquático um jogo desportivo praticado em todo o mundo há mais de um século, a necessidade de um perfil de elevada exigência a nível físico, técnico e tático é amplamente reconhecido pela comunidade científica. À semelhança de outros jogos desportivos, cada encontro é único e de desfecho imprevisível, sucedendo-se as ações de jogo de forma não linear no espaço e no tempo. A natureza complexa destes jogos é reconhecida por vários pesquisadores, sabendo-se que exige das equipas e dos jogadores capacidades específicas para responder à variabilidade e superar as dificuldades 
que vão surgindo (Melchiorri et al., 2020). Sendo o polo aquático um sistema complexo, organizado e de final aberto (Canossa et al., 2020), são múltiplos os fatores que de forma inter-relacionada nele intervêm. Pelo facto de se desenrolar no meio aquático a dimensão técnica é fundamental, sobretudo em idades mais baixas, para que através de um treino de qualidade os jogadores se possam preparar e atingir elevados níveis de prestação (Bratusa et al., 2006)

Neste sentido, na avaliação inicial do INEX, os jogadores de polo aquático mais velhos (coorte 3) mostraram ser tecnicamente mais evoluídos que os seus pares no salto vertical $\left(F=7.55, p<.05, \mathrm{p \eta}^{2}=.16\right)$, velocidade de nado $\left(F=13.39, p<.001, \mathrm{p \eta}^{2}=.26\right)$ e remate direto $\left(F=0.43, p<.05, \mathrm{pn}^{2}=.01\right)$. Por outro lado, os sujeitos da coorte 2 apresentaram melhores percentagens de eficácia nos três gestos técnicos de remate, isto é, após drible $\left(F=2.41, p<.05, \mathrm{p \eta}^{2}=.06\right)$ e de precisão direto $\left(F=3.13, p<.001, \mathrm{p \eta}^{2}=.07\right)$ e após drible $(F$ $=0.06, p<.05, \mathrm{p \eta}^{2}=.00$ ) (QUADRO 2). Sendo esperado que as idades mais novas sejam alvo da instrução e prática específica das habilidades motoras básicas (Malina \& Eisenman, 2009), o maior investimento em sessões de treino exclusivamente dedicadas à técnica individual dos gestos técnicos específicos do polo aquático a partir destas idades parece explicar os resultados obtidos. No entanto, o treino específico da precisão do remate, que deve ser uma realidade (Canossa et al., 2016), poderá não estar a acontecer nos jogadores da coorte 3. De facto, como estes jogadores são mais solicitados para integrarem as equipas que competem regularmente pode haver uma maior tendência para o de treino de outros aspetos específicos da preparação para os jogos.

Tratando-se de um jogo que requer não só habilidade de nado, mas também trabalho de equipa, força, resistência e agilidade (Gardasevic \& Joksimovic, 2020), a sua interdependência é evidente face à contextualização espácio-temporal em que o jogo se desenrola Nesse sentido, as habilidades táticas são também de elevada relevância (Viero et al., 2020) tendo-se observado que os jogadores mais velhos tinham mais agilidade na sua tomada de decisão aberta (movendo-se mais rápido). 0 facto de nestas idades se iniciar a vertente competitiva pode explicar a aquisição e desenvolvimento de capacidades preceptivas cognitivas que são exigidas e importantes para o desempenho no jogo, principalmente em ações defensivas rápidas e com grade capacidade de antecipação (Tucher et al., 2015) Também se verificou que os jogadores da coorte 3 tem maior potência aeróbia relativamente aos seus pares $\left(F=11.55, p<.001, \mathrm{p \eta}^{2}=.23\right)$, o que poderá justificar pelo aumento de tempo de treino e pela iniciação às atividades competitivas formais nestas idades. percetivo-cognitivas), por coorte, no baseline

\begin{tabular}{|c|c|c|c|c|c|}
\hline \multirow{3}{*}{$\begin{array}{l}\text { DOMÍNIO DAS HABILIDADES } \\
\text { ESPECÍFICAS/PERCETIVO- } \\
\text { COGNITIVAS }\end{array}$} & COORTE 1 (C1) & COORTE 2 (C2) & COORTE 3 (C3) & \multirow{3}{*}{$F\left(\mathrm{pn}^{2}\right)$} & \multirow{3}{*}{ CONTRASTE } \\
\hline & $(n=28)$ & $(n=41)$ & $(n=11)$ & & \\
\hline & $M \pm D P$ & $M \pm D P$ & $M \pm D P$ & & \\
\hline \multicolumn{6}{|l|}{ HABILIDADES TÉCNICAS } \\
\hline Salto vertical (cm) & $108.46 \pm 9.17$ & $116.39 \pm 9.04$ & $118.00 \pm 9.59$ & $7.55 \mathrm{~ns}(.16)$ & $\mathrm{C} 2<\mathrm{C} 3<\mathrm{C} 4$ \\
\hline Sprint (s) & $8.31 \pm 1.25$ & $7.12 \pm 0.72$ & $7.10 \pm 1.03$ & $13.39^{\star \star \star}(.26)$ & $\mathrm{C} 2>\mathrm{C} 3>\mathrm{C} 4$ \\
\hline Remate direto (\%) & $51.79 \pm 22.83$ & $53.66 \pm 29.70$ & $60.61 \pm 22.69$ & $0.43 n s(.01)$ & $\mathrm{C} 2<\mathrm{C} 3<\mathrm{C} 4$ \\
\hline Remate após drible (\%) & $43.45 \pm 30.88$ & $57.32 \pm 26.89$ & $45.45 \pm 15.08$ & $2.4 \operatorname{lns}(.06)$ & $\begin{array}{c}\mathrm{C} 2<\mathrm{C} 3<\mathrm{C} 4 ; \\
\mathrm{C} 3>\mathrm{C} 4\end{array}$ \\
\hline Remate de precisão direto (\%) & $16.67 \pm 16.97$ & $26.83 \pm 19.32$ & $18.18 \pm 8.99$ & $3.13^{\star}(.07)$ & $\begin{array}{c}\mathrm{C} 2<\mathrm{C} 3<\mathrm{C} 4 ; \\
\mathrm{C} 3>\mathrm{C} 4\end{array}$ \\
\hline Remate de precisão após drible (\%) & $21.43 \pm 14.24$ & $22.76 \pm 19.63$ & $21.21 \pm 13.10$ & $0.06 n s(.00)$ & $\mathrm{C} 2<\mathrm{C} 3>\mathrm{C} 4$ \\
\hline \multicolumn{6}{|l|}{ HABILIDADES TÁTICAS } \\
\hline Agilidade (s) & $5.77 \pm 0.75$ & $5.17 \pm 1.11$ & $4.64 \pm 0.90$ & $6.16 n s(.14)$ & $\mathrm{C} 2, \mathrm{C} 3>\mathrm{C} 4$ \\
\hline Potência aeróbia (m/s) & $0.92 \pm 0.03$ & $0.95 \pm 0.05$ & $1.00 \pm 0.08$ & $11.55^{\star \star *}(.23)$ & $\mathrm{C} 2, \mathrm{C} 3<\mathrm{C} 4$ \\
\hline
\end{tabular}

Nota: $p<.05$

\section{DOMÍNIO CONTEXTUAL}

Os fatores contextuais, embora influenciem de forma indireta a prestação dos jogadores de polo aquático, tem uma importância decisiva desde a etapa de formação, passando pela fase de especialização e indo até ao alto rendimento. Nesse sentido, de seguida iremos referir-nos a intervenientes decisivos no processo de treino desportivo dos jovens jogadores por nós estudados, uns que intervem diretamente nesse processo (os treinadores e os clubes) e outros que suportam a pratica desportiva como parte integrante da vida dos seus educandos (os pais).

PAIS

Muitas crianças e jovens referem que os seus familiares não reagem de forma positiva perante os seus êxitos e até os penalizam quando falham. Este comportamento propicia o "medo de falhar" pois o praticante percebe as consequências negativas advindas de momentos em que não tem êxito (Marin \& Abraldes, 2020). Embora esta conduta não seja 
específica do desporto, mas seja um comportamento social bastante comum quando os filhos não obtêm os resultados que os pais desejam, é importante melhor compreender o contexto no qual os jovens jogadores de polo aquático estão inseridos, o qual desempenha um papel importante no seu desenvolvimento e desempenho desportivo.

A informação obtida dos pais relativa à importância do desporto e à competência desportiva são de nível moderado a elevado, assim como no suporte e encorajamento para a prática desportiva, dedicando em média $5 \mathrm{~h}$ por semana às atividades dos filhos. 0 pape dos pais é importante na formação das atitudes e perceções de uma criança pois eles influenciam a perceção da criança sobre competência, orientação para objetivos e atribuições que a mesma faz sobre a sua habilidade (Welk et al., 2009). Informação detalhada relativa a esta temática poderá ser consultada no quadro 3.

QUADRO 3. Estatísticas da informação dos pais no polo aquático

\begin{tabular}{lll}
\hline PAIS $(N=$ 31) & $M \pm D P$ & MIN-MÁX \\
\hline Perceção da competência dos filhos & $4.04 \pm 0.11$ & $3.00-5.00$ \\
\hline Perceção do valor do desporto & $4.39 \pm 0.10$ & $3.00-5.00$ \\
\hline Suporte e encorajamento & $3.92 \pm 0.10$ & $2.85-4.77$ \\
\hline Tempo dedicado à prática desportivados filhos por semana (horas) & $5.44 \pm 4.86$ & $0.00-25.00$ \\
\hline
\end{tabular}

TREINADORES

O perfil do treinador de polo aquático dos escalões de formação é um tema muito interessante, mas também pouco estudado. É sabido que o processo de aprendizagem/treino de jovens é melhor conseguido quando os jogadores se sentem seguros (em controlo da situação), curiosos/interessados, admirados pela figura do treinador/dos pares e alegres. Neste contexto, todas as oportunidades são boas para experimentação, descoberta e consequente aprendizagem. Pelo contrário, o desenvolvimento de competências não se proporciona de forma tão eficaz quando o praticante tem medo/receio, sente inveja/culpa ou está triste. Felizmente, por ser um jogo coletivo e com bola, os treinadores de polo aquático tem um ambiente propicio ao desenvolvimento de competências pelo facto dos seus jogadores estarem intrinsecamente motivados. No entanto, devem alimentar essa motivação para a prática, nomeadamente através da variabilidade de estímulos e exercícios (Fernández \& García, 2018).

Os treinadores reportaram um nível de eficácia moderado a elevado, com a pontuação mais alta no caráter e a mais baixa na estratégia de jogo. Por outro lado, demonstraram uma variação entre as subescalas de comportamento do treinador, com pontuações mo- deradas a elevadas no treino e instrução e feedback positivo, e baixas a moderadas no apoio social, comportamento democrático e autocrático. Em média, tinham 10 anos de experiência como treinadores de polo aquático, sendo todos ex-desportistas. Dois possuíam o Grau 1 de treinador, três o Grau 2 e dois o Grau 3. Dos sete treinadores, apenas dois tiveram funções de selecionador regional e um de selecionador nacional (QUADRO 4).

QUADRO 4. Estatísticas da informação dos treinadores de polo aquático

\begin{tabular}{|c|c|c|c|}
\hline TREINADORES $(N=7)$ & $M \pm D P$ & MIN-MÁX & $n(\%)$ \\
\hline \multicolumn{4}{|l|}{ EFICÁCIA DO TREINADOR } \\
\hline Estratégia & $3.92 \pm 0.12$ & 3.43-4.29 & \\
\hline Motivação & $4.10 \pm 0.12$ & $3.71-4.71$ & \\
\hline Técnica de ensino & $4.28 \pm 0.16$ & $3.86-4.86$ & \\
\hline Caráter & $4.57 \pm 0.19$ & $3.75-5.00$ & \\
\hline \multicolumn{4}{|l|}{ COMPORTAMENTO DO TREINADOR } \\
\hline Treino e instrução & $3.98 \pm 0.13$ & $3.62-4.46$ & \\
\hline Comportamento democrático & $2.68 \pm 0.19$ & $2.11-3.67$ & \\
\hline Comportamento autocrático & $2.23 \pm 0.08$ & $2.00-2.60$ & \\
\hline Apoio social & $2.87 \pm 0.25$ & $2.00-4.13$ & \\
\hline Feedback positivo & $4.00 \pm 0.21$ & $3.20-5.00$ & \\
\hline \multicolumn{4}{|c|}{ INFORMAÇÃO DEMOGRÁFICA E DESPORTIVA } \\
\hline Experiência como treinador (anos) & $10.57 \pm 1.90$ & $3.00-20.00$ & \\
\hline \multicolumn{4}{|l|}{ Grau de treinador } \\
\hline Grau 1 & & & $2(28.57)$ \\
\hline Grau 2 & & & $3(42.86)$ \\
\hline Grau 3 & & & $2(28.57)$ \\
\hline Ex-atleta (sim/não) & & & $7(100) / 0(0)$ \\
\hline Treinador da seleção regional (sim/não) & & & $2(28.57) / 5(71.43)$ \\
\hline Treinador da seleção nacional (sim/não) & & & $1(14.28) / 6(85.71)$ \\
\hline
\end{tabular}


CLUBES

Tradicionalmente, os clubes de polo aquático (ou com secções de polo aquático) portugueses têm recursos escassos. A juntar a esse facto, os horários disponíveis para treino, mesmo dos escalões jovens, são em momentos tardios do dia (já de noite), normalmente após as equipas de natação pura desportiva terminarem as suas atividades (após as $21 \mathrm{~h}$ ). Ao jogador de polo aquático é exigido "saber nadar" num contexto específico que é o campo de jogo e a sua formação terá de passar pelo domínio do espaço. Nesse sentido, a informação recolhida sobre os clubes e as condições que propiciam a prática é muito importante.

A informação recolhida sobre os clubes mostra uma variação substancial entre eles, nomeadamente no número de modalidades desportivas disponibilizadas, número de praticantes de todas as modalidades em gera,l e de polo aquático em particular, escalões competitivos que contemplados, quantidade de títulos nacionais e regionais por clube e há quantos anos existia a secção de polo aquático. Nenhum clube (que tenha respondido aos respetivos questionários) possuía as suas próprias instalações (sendo posse da respetiva autarquia, na sua maioria), metade dos clubes possuía ginásio, $75 \%$ dispunha de gabinete médico/fisioterapia, um possuía área de aquecimento e nenhum possuía sala de vídeo e hidroterapia. 0 número de treinadores de polo aquático por clube variava entre dois e seis, tendo a maior parte formação de Grau 1 e 2 (o que poderá implicar um nível menos elevado na qualidade da transmissão de conteúdos, apoio social e orientação tático-estratégica das equipas). Complementarmente, observa-se que a maioria dos clubes tinha fisioterapeuta, metade tinha médico, um tinha nutricionista, mas nenhum tinha psicólogo (que nestas idades é fundamental no apoio à atividade, integração do jovem jogador no grupo e resolução de conflitos inerentes à prática desportiva; Canossa et al., 2020). Todos os clubes utilizavam as redes sociais e um dispunha inclusivamente de estação de rádio ou canal de tv/online (QUADRO 5).

QUADRO 5. Estatísticas da informação dos clubes de polo aquático

\begin{tabular}{|c|c|c|c|}
\hline CLUBES $(N=6)$ & $M \pm D P$ & MIN-MÁX & $n(\%)$ \\
\hline \multicolumn{4}{|l|}{ CARACTERÍSTICAS } \\
\hline Número de modalidades & $6.00 \pm 3.16$ & $3-10$ & \\
\hline Número total de atletas & $464.00 \pm 516.34$ & $100-1200$ & \\
\hline Número de jogadores de polo aquático & $75.25 \pm 40.80$ & $40-134$ & \\
\hline Número de escalões competitivos & $6.25 \pm 0.50$ & $6-7$ & \\
\hline Número de títulos nacionais & $14.50 \pm 16.21$ & $1-36$ & \\
\hline Número de títulos regionais & $7.25 \pm 9.36$ & $0-21$ & \\
\hline Número de anos da secção de futebol & $20.25 \pm 12.42$ & $6-35$ & \\
\hline
\end{tabular}

\begin{tabular}{|c|c|c|c|}
\hline CLUBES $(N=6)$ & $M \pm D P$ & MIN-MÁX & $n(\%)$ \\
\hline \multicolumn{4}{|l|}{ INFRAESTRUTURAS } \\
\hline Instalaçōes próprias (sim/não) & & & $0(0.00) / 4(100.00)$ \\
\hline \multicolumn{4}{|l|}{ Equipamentos complementares } \\
\hline Ginásio (sim/não) & & & $2(50.00) / 2(50.00)$ \\
\hline Área de aquecimento (sim/não) & & & $1(25.00) / 3(75.00)$ \\
\hline Gabinete médico/fisioterapia (sim/não) & & & $3(75.00) / 1(25.00)$ \\
\hline Hidroterapia (sim/não) & & & $0(0.00) / 4(100.00)$ \\
\hline Sala de vídeo (sim/não) & & & $0(0.00) / 4(100.00)$ \\
\hline Treinos sempre nas instalações do clube (sim/não) & & & $3(75.00) / 1(25.00)$ \\
\hline \multicolumn{4}{|l|}{ RECURSOS HUMANOS } \\
\hline Número de treinadores de polo aquático & $4.00 \pm 1.83$ & 2-6 & \\
\hline \multicolumn{4}{|l|}{ Nível de certificação dos treinadores } \\
\hline Número de treinadores com Grau 1 & $1.50 \pm 1.00$ & $0-2$ & \\
\hline Número de treinadores com Grau 2 & $1.50 \pm 1.29$ & $0-3$ & \\
\hline Número de treinadores com Grau 3 & $1.00 \pm 0.82$ & $0-2$ & \\
\hline \multicolumn{4}{|l|}{ STAFF } \\
\hline Médico (sim/não) & & & $2(50.00) / 2(50.00)$ \\
\hline Psicólogo (sim/não) & & & $0(0.00) / 4(100.00)$ \\
\hline Fisioterapeuta (sim/não) & & & $3(75.00) / 1(25.00)$ \\
\hline Nutricionista (sim/não) & & & $1(25.00) / 3(75.00)$ \\
\hline \multicolumn{4}{|l|}{ COMUNICAÇÃO } \\
\hline Redes sociais (sim/não) & & & $4(100.00) / 0(0.00)$ \\
\hline Estação de rádio ou canal de tv/online (sim/não) & & & $1(25.00) / 3(75.00)$ \\
\hline
\end{tabular}


O presente estudo mostra que os jogadores mais velhos, relativamente aos seus pares, são mais pesados, tem valores superiores de massa isenta de gordura e são maturacionalmente mais avançados. Revelam também melhores valores das variáveis relacionadas com a força muscular do tronco e dos membros superiores (provavelmente devido ao treino específico e à exigência do jogo quanto à propulsão no meio aquático). Os mesmos sujeitos são igualmente mais evoluídos quanto à potência aeróbia e tomada de decisão aberta bem como quanto ao salto vertical, velocidade de nado e remate direto (embora menos eficazes neste gesto técnico). É reconhecida a importância do desporto e da competência desportiva pelos pais, encorajando e dando suporte à prática dos seus filhos. Os treinadores parecem desempenhar adequadamente o seu papel, embora tenham reportado um nível baixo quanto à estratégia de jogo, o que poderá ser reflexo da baixa formação dos treinadores dos jovens jogadores. Refere-se, por último, que as características dos clubes, staff e recursos humanos são muito variáveis (exceto o facto das instalações não serem próprias). A maioria das piscinas são camarárias, o que também contribui para a alocação de horários menos favoráveis à prática do polo aquático em escalões de formação.

\section{IMPLICAÇÕES PARA O TREINADOR}

1. Que condições de prática favorecem o desenvolvimento sustentável do jogador de polo aquático?

As condições que vão de encontro à organização social e desportiva do país, usualmente considerada favorável, como sejam a atribuição de horários de prática compatíveis com a atividade escolar dos jovens. Tal implica que as sessões de treino não deveriam ocorrer em horas tardias, condições que favoreceriam o apoio parental da prática.

2. 0 que se entende por desenvolvimento sustentável do jogador de polo aquático a longo prazo?

0 processo de ensino e treino que procure ir de encontro às necessidades de desenvolvimento psico-motor do indivíduo, desde muito jovem, enaltecendo a sua evolução e conquistas, num ambiente de treino favorável que prime pelo aspeto lúdico da prática.

3. O que se espera dos treinadores no plano da preparação dos jovens jogadores de polo aquático? Que se comprometam com o desenvolvimento sustentável a longo prazo dos praticantes, disponibilizando oportunidades similares para todos, segundo um plano previamente traçado. Que planeiem a atividade de acordo com o escalão/nível dos jogadores e invistam na sua própria formação para que estejam aptos a melhor formar.
A aquisição de princípios básicos do jogo em que a tomada de decisão criativa seja potenciada, dentro do plano da equipa. 0 desenvolvimento dos requisitos motores básicos que permitam ao praticante enfrentar desafios e orquestrar, partilhar ou participar em projetos delineados com os companheiros.

5. E qual o papel que os quadros competitivos poderão ter neste processo?

Competir a um nível mais elevado tem um efeito potenciador dos níveis técnico-táticos e físico-atléticos dos jovens jogadores, para além de ser motivador e estimulante. Porém, tal requer ajustamentos do plano de treino, periodização e monotorização da prestação do jogador. 


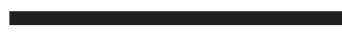

AUTORES:

Patrícia Coutinho

Ana Ramos ${ }^{1}$

Cristiana Bessa ${ }^{1}$

Beatriz Valongo

José Afonso ${ }^{1}$

Isabel Mesquita ${ }^{1}$

Centro de Investigação, Formação, Intervenção e Inovação em Desporto

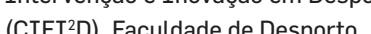
(CIr.

\section{https://doi.org/10.5628/rpcd.21.S2.121}

Gardasevic, N. \& Joksimoviv, M., (2020). The impact of the change of water polo rules on the game dynamics. international Journal of Physical Education, Fitness and Sports, 9(2), 51-56.

Marin, L. C., \& Abraldes, J. A. (2020). Gestión y formación en un club desportivo: Rendimiento y motivación. In J. A Abraldes, R. J. Fernandes, F. A. Castro (Eds.), Waterpole Test de valoración del rendimento deportivo (pp. 175-199) Diego Marin Librero-Editor.

Melchiorri, M., Viero, V., Tancredi, V., Bianco, R. \& Bonifazi, M. (2020). Actual playing time of water polo players in relation to the field position. Journal of Human Kinetics, 73, 241-249.

Tucher, G., Castro, F., Silva, A., \& Garrido, N. (2015). The Functional Test for Agility Performance is a reliable quick decision-making test for skilled water polo players. Journal of Human Kinetics, 46, 157-165.

Viero, V., Triossi, T., Bianchi, D., Campagna, A. \& Melchiorri G. (2020). Physical and performance variables for talent identification in water polo. The Journal of Sport Medicine and Physical Fitness, 60,1309-1316.

Welk, G., Babkes, M. \& Schaben, J. (2009). Parental influences on youth sport participation. In M. Coelho e Silva, A. Figueiredo, M. Elferink-Gemser, \& R. Malina (Eds.), Youth sports: Participation, trainability and readiness (pp. 75103). Imprensa da Universidade de Coimbra.

\section{DOMÍNIO BIOLÓGICO}

No início era assim.

\section{Unidade na diversidade.}

Voleibol

Atualmente, o processo de preparação de um jovem atleta e a elaboração dos respetivos programas de treino pressupõem a existência de um conhecimento teórico e prático sólido, proveniente de vários domínios das Ciências do Desporto. Informação relevante sobre indicadores de controlo do processo de treino, como, por exemplo, as características físicas dos jogadores, os seus atributos fisiológicos e as suas competências tático-técnicas, podem ser efetivamente refletidos e aplicados em programas de treino com o intuito de desenvolver e potenciar a performance do jovem atleta (Lidor \& Ziv, 2010). Em conformidade, diversos estudos apresentam uma relação entre as características antropométricas e de composição corporal do jogador de voleibol e a sua performance a nível físico e técnico (Duncan et al., 2006; Lidor \& Ziv, 2010; Milic et al., 2017). Assim, importa dar a conhecer o perfil do jovem jogador de voleibol português para que os treinadores possam preparar e aferir com mais rigor e qualidade os processos de preparação dos atletas e, consequentemente, potenciar o seu desenvolvimento e a sua performance a médio e longo prazo.

No momento da primeira avaliação (baseline) observaram-se diferenças significativas entre os voleibolistas com mais idade (coorte 4) e os seus pares mais novos (coortes 1-3) Especificamente, os jogadores com mais idade eram mais altos $\left(F=17.35, p<.001, \mathrm{p \eta}^{2}\right.$ $=.32)$, mais pesados $\left(F=8.79, p<.001, \mathrm{p \eta}^{2}=.19\right)$, apresentaram maior envergadura $(F=$ $\left.11.72, p<.001, \mathrm{p \eta}^{2}=.24\right)$, diâmetro palmar transversal $\left(F=14.69, p<.001, \mathrm{p \eta}^{2}=.28\right)$ e longitudinal $\left(F=14.91, p<.001, \mathrm{pn}^{2}=.29\right)$, e, ainda, mais massa isenta de gordura $(F=$ $15.40, p<.001, \mathrm{pn}^{2}=.30$ ) (QUADRO 1). Foram igualmente encontradas diferenças significa- 